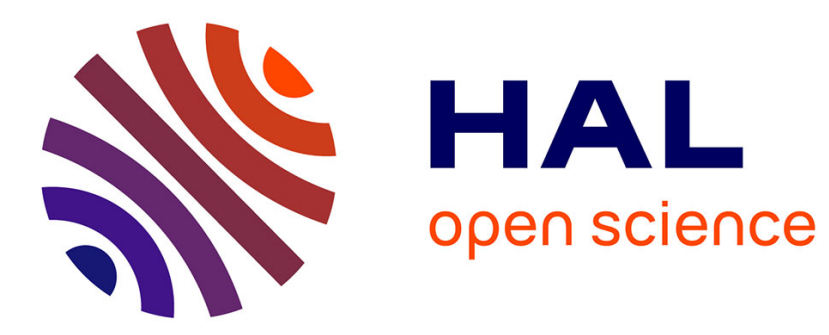

\title{
A Qualitative Theory of Conflict Resolution and Political Compromise
}

\author{
Joseph M. Abdou, Hans Keiding
}

\section{To cite this version:}

Joseph M. Abdou, Hans Keiding. A Qualitative Theory of Conflict Resolution and Political Compromise. 2018. hal-01796062

\section{HAL Id: hal-01796062 \\ https://hal.science/hal-01796062}

Preprint submitted on 19 May 2018

HAL is a multi-disciplinary open access archive for the deposit and dissemination of scientific research documents, whether they are published or not. The documents may come from teaching and research institutions in France or abroad, or from public or private research centers.
L'archive ouverte pluridisciplinaire HAL, est destinée au dépôt et à la diffusion de documents scientifiques de niveau recherche, publiés ou non, émanant des établissements d'enseignement et de recherche français ou étrangers, des laboratoires publics ou privés. 


\title{
A Qualitative Theory of Conflict Resolution and Political Compromise
}

\author{
Joseph Abdou* Hans Keiding ${ }^{\dagger}$
}

April 18, 2018

\begin{abstract}
We view political activity as an interaction between forces seeking to achieve a political agenda. The viability of a situation depends on the compatibility of such agendas. However even in a conflictual situation a compromise may be possible. Mathematically a political structure is modeled as a simplicial complex and a viable configuration as a simplex. A represented compromise is a viable configuration obtained by the withdrawal of some agents in favor of some friendly representatives. A delegated compromise is a sophisticated version of a compromise obtained by the iteration of the withdrawal process. Existence of such solutions depends on the discrete topology of the simplicial complex. In particular we prove that the existence of a delegated compromise is equivalent to the strong contractibility of the simplicial complex.
\end{abstract}

Keywords: Delegation, compromise, simplicial complex, contiguity, strong homotopy.

JEL Classification: C70, C79, AMS Classification: 91A70.

\section{Introduction}

We model political activity as an interaction between forces seeking to achieve a political agenda. A stable government allows the implementation of the political agenda of the party or the coalition holding power. In normal circumstances, the struggle for power is regulated by constitutional rules that guarantee a peaceful and consensual outcome of the process. This includes commonly admitted mechanisms for breaking deadlocks once they occur (for instance elections, referendum or justice ruling). However many

\footnotetext{
${ }^{*}$ Centre d'Economie de la Sorbonne, Université Paris 1, Panthéon-Sorbonne, 106-112 boulevard de l'Hôpital, 75647 Paris Cedex 13-France; email: abdou@univ-paris1.fr.

${ }^{\dagger}$ Department of Economics, University of Copenhagen, O.Farimagsgade 3, DK-1353 Copenhagen K, Denmark; email: hans.keiding@econ.ku.dk.
} 
political entities, under special circumstances, incur a blocked governance process while the current order fails to provide a clue for a solution. This is a political crisis or a stalemate. For instance, one can witness the formation of a crisis in a place where a military conflict left the entity with a pre-state, pre-constitutional configuration, that is, where a universally accepted rule does not exist; or where the threat of a violent action impedes the regular unfolding of the political process; or else where the risk of disrupting the ongoing process is wielded by a force that accumulates discontent with the current establishment.

The general question that we ask is therefore : What happens in a crisis configuration that is unsolvable by the current institutions, or put more explicitly what can be expected if a configuration composed by many parties with incompatible political agendas lacks the institutional mechanism that enforces a settlement?

Modeling politics has a long history starting from the early greek philosophers. One of the main concepts that marked the modern analysis of conflict is the notion of enemy. According to political thinkers that promote this view the essential moment of politics is the discrimination friend/enemy (cf. e.g.[11]). This binary choice erupts in a situation of disruption, when the political body is in danger, war or civil strife. In this model we admit that political action is by essence produced in a disrupted situation, that is a preinstitutional moment, but we depart from the idea that politics is by essence bi-polar: political action is not drawing a line of separation from (or the destruction of) an enemy but the search of a viable situation from a disrupted one. Political forces in presence can be either compatible for an inclusive governance or not, but this compatibility concerns each subgroup of forces. The current situation is therefore described by this compatibility relation. Mathematically a configuration is represented by a simplicial complex.

The fundamental notion in our approach to crisis resolution in simplicial complexes is delegation: An agent can delegate power to another agent when the latter is at least as well situated in the power hierarchy as the delegating agent. The result of delegation will be a power structure which is simplified to some extent, since some of the sources of incommensurability have been removed. We investigate in detail what can emerge as a result of delegation, giving rise to the notion of representations and represented compromises, which in particular cases may resolve the instabilities fully. Turning next to cases of iterated delegation, where delegation may result in loss of influence as the power originally handed over to another agent is then further delegated. On the other hand, such repeated delegation increases the possibility of avoiding stalemates in decision making.

From a formal point of view, the model falls within the field of discrete topology; both the representations and the delegations can be studied using the theory of homotopy in finite simplicial complexes, and the relevant concepts are introduced when when needed. 
The paper is organized as follows: In the following Section 2, we introduce the basic notion of a political structure which has the the mathematical structure of a simplicial complex, and we define the notion of a delegation, giving rise to representations and represented compromises. As we proceed, examples show how these notions apply to simple games and to network models of communication. In the following Section 3, the formalism is developed somewhat further so we can use results from homotopy theory of finite complexes to investigate delegation in political structures. The main results on the structure of represented compromises are presented in Section 4, which also points to some of the shortcomings. This leads to the consideration of the somewhat stronger form of delegation and its consequences, the delegated compromises, in Section 5. Finally, Section 6 contains some concluding comments.

\section{Basic definitions}

We consider a situation described by a nonempty set $E$ of agents. In the interpretation, agents may be individuals, but it may as well be groups of individuals or even political issues or institutions. A nonempty subset of agents is called a configuration. We assume that there is given a set $\mathcal{K}$ of viable configurations, with the property that any singleton $\{x\}$, where $x \in E$, is viable, and any non empty subset of a viable configuration is viable, so that formally $\mathcal{K}$ is a simplicial complex, with set of vertices $E$. The pair $(E, \mathcal{K})$ is called a political structure.

Corresponding to what was said about agents, the interpretation of viable configuration may vary according to the circumstances. In general, viability represents a form of compatibility between agents, whereas configurations which are not viable will display some sort of conflict preventing the functioning of the system. Participation in viable configurations gives a first notion of the importance of an agent in the overall structure.

In the sequel the power set of any set $X$ will be denoted $\mathcal{P}(X)$, and $\mathcal{P}(X) \backslash\{\emptyset\}$ will be denoted $\mathcal{P}_{0}(X)$.

Example 1 (TU games). A TU (Transferable Utility) game is a pair $(N, v)$, where $N$ is a nonempty set of players and $v$ is a map from the set $\mathcal{P}_{0}(N)$ of nonempty subsets of $N$ (coalitions) a number interpreted as the money or utility gain that the coalition can obtain for its members. An payoff vector in $(N, v)$ is a vector $x \in \mathbb{R}^{N}$ satisfying $\sum_{i \in N} x_{i} \leq v(N)$; let $V(N)$ be the set of payoff vectors. For $S \in \mathcal{P}_{0}(N)$, let $A(S)=$ $\left\{x \in V(N) \mid \sum_{i \in S} x_{i} \geq v(S)\right\}$ be the set of payoff vectors which cannot be improved by the coalition $S$, in the sense its members get at least as much as it they could get from the coalition. A family of coalitions is viable if there is a payoff vector which cannot be improved by any coalition in the family. A political structure $\left(\mathcal{P}_{0}(N), \mathcal{K}_{v}\right)$ is defined 
by positing :

$$
\mathcal{K}_{v}=\left\{\sigma \subseteq \mathcal{P}_{0}(N) \mid \cap_{S \in \sigma} A(S) \neq \emptyset\right\} .
$$

Note that $A(S) \neq \emptyset$ and therefore the set of vertices is indeed $\mathcal{P}_{0}(N)$. Moreover $\mathcal{P}_{0}(N)$ belongs to $\mathcal{K}_{v}$ if and only if there is a payoff vector which cannot be improved by any coalition, equivalently if and only if the core of $(N, v)$ is nonempty. A closer study of $\mathcal{K}_{v}$ may however be helpful in the nontrivial case where $\mathcal{P}_{0}(N) \notin \mathcal{K}_{v}$. It should be noticed that here the agents are coalitions, and configurations are families of coalitions.

Example 2 (Effectivity structures). Let $N$ and $A$ be finite nonempty sets (of agents and alternatives, respectively). An effectivity structure on $(N, A)$ is a subset of $\mathcal{P}_{0}(N) \times$ $P_{0}(A)$, so that elements of $E$ are pairs $(S, B)$ with $S \subseteq N, B \subseteq A$. In the interpretation, elements of $E$ are potential cases of exercising power: the coalition $S$ can make sure that outcome of any decision made in the community must belong to $B$, or equivalently, $S$ can prevent choices outside $B$. One can consider $E$ as a (partially defined) effectivity function: that is $E: \mathcal{P}_{0}(N) \rightarrow \mathcal{P}\left(\mathcal{P}_{0}(A)\right)$ where $E(S)$ stands for $\left\{B \in \mathcal{P}_{0} A \mid(S, B) \in E\right\}$ (cf Abdou and Keiding [1]).

In order to make of $E$ a political structure with elements $(S, B)$ as agents we have to describe viable configurations. For that purpose we need to specify the environment in which the power expressed by $(S, B)$ may be exercised: Let $\mathcal{L}(A)$ denote the set of linear orders on $A$, and define (preference) profiles as maps $R: N \rightarrow \mathcal{L}(A)$, so that the set of profiles is $\mathcal{L}(A)^{N}$. If for some $i \in N$, the alternative $a$ is (strictly) preferred to the alternative $b$ in the profile $R$, then we write $a R_{i}^{+} b$, and for $a \in A$, the set of alternatives preferred to $a$ by all members of $S$ in the profile $R$ is

$$
P(a, S, R)=\left\{b \in A \mid b R_{i}^{+} a, \text { all } i \in S\right\} .
$$

With this notation, we get that the coalition $S$ is induced to exercise its objection power at the profile $R$ if an alternative $c \in A$ is suggested such that $P(c, S, R)$ contains a subset $B$ with $(S, B) \in E$. We shall say that a configuration $s \subseteq E$ is viable if it is always able to select an alternative against which none of its members will exercise their objection power, that is if for each profile $R$ there is some $a \in A$ such that $B$ is not contained in $P(a, S, R)$ for some $(B, S) \in s$. Considering the configuration $s \subseteq E$ as an effectivity function, viability of $s$ is equivalent to stability of $s$ as considered by e.g. Moulin and Peleg [4], Abdou and Keiding [1]. It is easy to see that the family $\mathcal{K}$ of all viable configurations in $E$ is indeed a simplicial complex, so that the ordered pair $(E, \mathcal{K})$ can be considered as a political structure.

Since our overall purpose is the investigation of conflictual situations and their possible resolution, we are mainly interested in ways in which to eliminate non-viable 
configurations. Our approach will be to allow agents to delegate their influence to other, more centrally placed agents. In our present setup we formulate this by the notion of delegation.

Definition 1. Let $y \in E$ and $d \subseteq E, y \notin d$. A delegation from $d$ to $y$ is a map $\delta_{d}: E \rightarrow E$ such that $\delta_{d}(x)=y$ if $x \in d$ and $\delta_{d \rightarrow y}(x)=x$ if $x \notin d$. A simple delegation is a delegation $\delta_{d \rightarrow y}$ where $d$ is a singleton, $d=\{x\}$, and it is written as $\delta_{x \rightarrow y}$.

A delegation $\delta_{d \rightarrow y}$ is said to be friendly if $s \cup\{y\} \in \mathcal{K}$ for all $s \in \mathcal{K}$ such that $s \cap d \neq \emptyset$.

A delegation from $d$ to $y$ can be seen as a political action by which the agents in $d$ withdraw from the political interaction in favor of the agent $y$. A delegation is friendly whenever any agent in $d$ can ensure that every viable configuration to which she participates remains viable after delegation, and moreover, the agent receiving the delegation is present in every configuration which contained the delegating agent. A withdrawal in favor of a delegate is more likely to happen if the delegation is friendly. In our analysis, the possibility of friendly delegations constitutes the only driving principle behind potential moves that reduce conflicts in a political structure.

Example 3 (Networks). The use of formal networks has a long history in the social sciences, with its beginning in the 1930s; for a survey of its history, see e.g. [12]. A social structure is a graph $\mathcal{G}=(V, E)$, where $V$ is a finite set of edges and $E$ is a set of two-element subsets $\left\{v_{0}, v_{1}\right\}$ of $E$. In the interpretations, $V$ are individuals, and two individuals $v_{0}, v_{1}$ are socially connected if $\left\{v_{0}, v_{1}\right\} \in E$. A graph is a simplicial complex where simplices have dimension at most 1 .

The social contacts is represented by a paths in the graph, that is sequences $\left\{v_{0}, v_{1}\right\}$, $\left\{v_{1}, v_{2}\right\}, \ldots,\left\{v_{n-1}, v_{n}\right\}$ of edges in $\mathcal{G}$. If there is a path from $v_{0}$ to $v_{n}$, then $v_{0}$ is indirectly socially connected to $v_{n}$, and the distance of this indirect connection is the shortest distance of a path from $v_{0}$ to $v_{n}$. In addition, the social position of an individual $v \in V$ can be measured by the degree of $v$, the number of edges in $E$ containing $v$. In Fig. 1 the individual represented by the point $v_{0}$ with degree 4 is clearly more centrally positioned than the other individuals. Centrality measures play an important role in network analysis, cf. [5].

There is a simple friendly delegation from $v_{0}$ to $v_{1}$ if any social relation of $v_{0}$ must be with $v_{1}$, so that $v_{1}$ is the only social contact of $v_{0}$. Cionsequently, an R-compromise is therefore a subset $V^{\prime}$ of $V$ such that all $v \in V \backslash V^{\prime}$ has degree 1. A pure R-compromise will exist only in specially simple networks. However, an extended version of the compromise, to be introduced later, will be more useful.

The following property of delegations is immediate. 


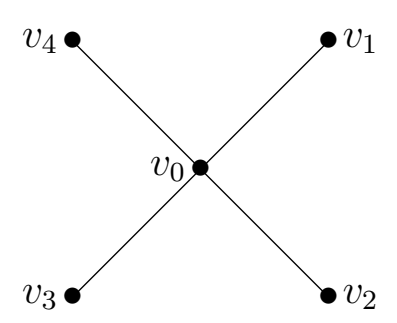

Figure 1: Point centrality in a network: The vertex $v_{0}$ with degree 4 is connected to all the other vertices with an edge, whereas the other vertices are connected only to $v_{0}$.

Proposition 1. For any $\emptyset \neq d=\left\{x_{1}, \ldots, x_{n}\right\} \subset E$ and $y \notin d$, if $\delta_{d \rightarrow y}$ is a delegation, then

$$
\delta_{d \rightarrow y}=\delta_{x_{1} \rightarrow y} \circ \cdots \circ \delta_{x_{k} \rightarrow y} .
$$

Moreover, $\delta_{d \rightarrow y}$ is friendly if and only if $\delta_{x_{j} \rightarrow y}$ is friendly for $j \in\{1, \ldots, n\}$.

As a result of the delegations, there may be fewer agents with conflicting interests. We introduce the notion of a compromise is as a counterpart of real world solutions to political conflicts, where agents (or issues, or power groups) will have to step aside since they will not be accepted as partners in a political deal but on the other hand can be represented by other, more acceptable, agents.

Definition 2. A nonempty subset $F$ of $E$ is a representation of $E$ if for any $x \notin F$, there exists some $y \in F$ such that $\delta_{x \rightarrow y}$ is a friendly delegation.

The subset $F$ of $E$ is a represented compromise (shorthand: an $R$-compromise) for $E$ if $F$ is a representation and $F \in \mathcal{K}$. A pure $R$-compromise is an element $y \in E$ such that $\{y\}$ is an R-compromise.

Example 4 (Empirical case). The recent stalemate in Lebanese politics and its resolution through compromise provides an illustration of the concepts introduced above. Beyond the formal constitution that was agreed upon in Taef in October 1989, the political configuration in Lebanon, in its principal components, that prevailed after the assassination of Prime Minister Rafic Hariri February 2005, can be described by the simplicial complex of Fig.2.

The polarizing issue that divided political forces can be described schematically as East-West, and compatibility of the agendas was dictated by that issue. Independently of that issue, the National Pact, partially represented by Taef Agrement, requires the effective inclusion in the governance of the three major confessions.

The major forces in presence in the conflict are the Future Movement (FUT), AMAL (AMA), Hizbullah (HIZ), Free Patriotic Movement (FPM), and Lebanese Forces (LF). In Fig.2, brown color has been assigned to Christians (Maronites), blue to Sunnis, and 


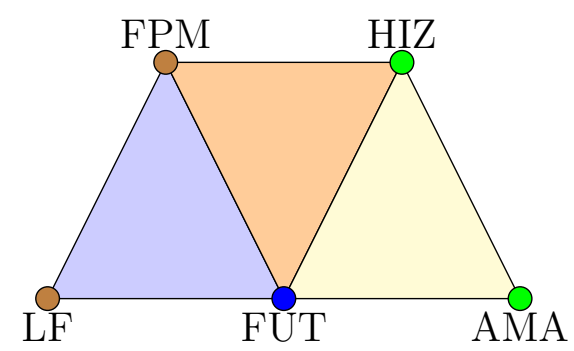

Figure 2: Friendly delegation and political compromise in Lebanese politics

green to Shias. After a long search, a compromise was found that respects the National Consensus, as represented by the middle shaded triangle. Presumably this compromise is the result of a friendly delegation from LF to either FPM or FUT, and from AMA to either FUT or HIZ. This compromise was formally implemented by the election of Michel Aoun as President (October 2016) and the formation of a goverment by Saad Hariri (December 2016).

In the following we shall study delegation and compromises using tools of finite homotopy theory, and for this purpose it is convenient to considered delegation from another angle, emphasizing that agents are removed from direct participation.

Definition 3. Let $\emptyset \neq F \subset E$. A retraction to $F$ is a map $r: E \rightarrow E$ such that $r(E)=F$ and $r(x)=x$ for all $x \in F$, and $F$ is said to be a retract if there is a retraction to $F$.

A retraction $r$ is friendly if $x \in s \in \mathcal{K}$ implies $\{r(x)\} \cup s \in \mathcal{K}$, and a friendly retract is a retract $F$ such that there exists a friendly retraction to $F$.

A retraction is a political action by which the conflictual political structure $(E, \mathcal{K})$ is reduced to the (sub) configuration $\left(F, \mathcal{K}_{F}\right)$, withdrawing agents not in $F$ in favor of suitable agents in $F$, their representatives. A retraction is friendly if any viable configuration containing a retracting agent as a member remains viable when he is replaced by his representative. Clearly, a delegation $\delta_{d \rightarrow y}$ is a retraction to $E \backslash d$, and a retraction $r$ to $F$ such that $r(E \backslash F)=\{y\}$ is a delegation from $E \backslash F$ to $y$. We have the following characterization:

Proposition 2. $F \subset E$ is a representation if and only if $F$ is a friendly retract.

Proof: Let $F$ be a representation. Define a map $r: E \rightarrow E$ as follows: $r$ is the identity on $F$, and if $x \notin F$ then put $r(x)=y$ where $y$ is some element of $F$ such that there is a friendly delegation from $x$ to $y$. It is clear that $r$ is a retraction on $F$. Moreover if $x \notin F$ for any $s \in \mathcal{K}$ such that $x \in s$, we have that $\{r(x)\} \cup s \in \mathcal{K}$; and for any $x \in F$ and $s \in \mathcal{K}$ such that $x \in s$, we have that $\{r(x)\} \cup s=s \in \mathcal{K}$. The converse is straightforward. 
The notion of friendly retraction characterizes a representation as the result of withdrawal of some agents, in what follows we consider whether a representation can result from of a sequential process of delegations.

Definition 4. A progressive delegation is a sequence $\delta_{x_{1} \rightarrow y_{1}}, \ldots, \delta_{x_{p} \rightarrow y_{p}}$ of simple delegations where the elements $x_{1}, \cdots, x_{p}$ are distinct and $y_{k} \notin\left\{x_{1}, \ldots, x_{k}\right\}$ for all $k=$ $1, \ldots, p$. A progressive delegation is friendly if each of the simple delegations that compose it is friendly. The configuration $c=\delta_{x_{p} \rightarrow y_{p}} \circ \cdots \circ \delta_{x_{1} \rightarrow y_{1}}(E)$ is called the outcome of the progressive delegation.

Thus, when performing a progressive delegation, agents are successively delegating their influence to other agents, and in this case it seems natural that once an agent has renounced on influence through delegation, she cannot herself be an object of delegation.

Proposition 3. Let $\delta_{x_{1} \rightarrow y_{1}}, \ldots, \delta_{x_{p} \rightarrow y_{p}}$ be a progressive delegation with $\phi=\delta_{x_{p} \rightarrow y_{p}} \circ$ $\cdots \circ \delta_{x_{1} \rightarrow y_{1}}$. Then $\phi$ is a retraction to $E \backslash\left\{x_{1}, \ldots, x_{p}\right\}$, and

$$
\operatorname{fix}_{\phi}=\{x \in E \mid \phi(x)=x\}=\phi(E)=E \backslash\left\{x_{1}, \ldots, x_{p}\right\} \neq \emptyset \text {. }
$$

Conversely, any retraction $\phi: E \rightarrow E$ to some $F \subseteq E$ can be obtained through a progressive delegation. Moreover all the involved delegations can be chosen to be friendly if and only if the retraction is friendly.

Proof: (i) Put $F=E \backslash\left\{x_{1}, \ldots, x_{p}\right\}$. It is clear that $F \subseteq$ fix $_{\phi}$. Conversely, for any $k \in\{1, \ldots, p\} \phi\left(x_{k}\right)=y_{\ell}$ with $\ell \geq k$ and by progressivity, $y_{\ell} \neq x_{k}$, so that $\operatorname{fix}_{\phi} \subset F$ and consequently fix $_{\phi}=F$.

Now, $F \subset \phi(E)$. In order to prove the opposite inclusion, let $x \in E$. Then either $x \in F$ and therefore $\phi(x)=x \in F$, or $x=x_{k}$ and $\phi(x)=y_{\ell}$ for $\ell \geq k$. It follows that $y_{\ell} \neq x_{k^{\prime}}$ for $k^{\prime} \geq \ell$ and by progressivity of the sequence $y_{\ell} \neq x_{k^{\prime}}$ for $k^{\prime}<\ell$ and finally $y_{\ell} \in F$. We conclude that $F=\phi(E)$.

For any $k \in\{1, \ldots, p\}$ let $\phi_{k}=\delta_{x_{k} \rightarrow y_{k}} \circ \cdots \circ \delta_{x_{1} \rightarrow y_{1}}$ and $F_{k}=E \backslash\left\{x_{1}, \ldots, x_{k}\right\}$. By convention $F_{0}=E$ and $\phi_{0}=\operatorname{Id}_{E}$. Then, by the first part of the proof $\phi_{k}$ is a retraction onto $F_{k}$ and one has $\phi_{k}=\delta_{x_{k} \rightarrow y_{k}} \circ \phi_{k-1}$. We prove by induction that $\phi_{k}$ is friendly. Clearly $\phi_{0}=\operatorname{Id}_{E}$ is friendly. Assume that $\phi_{k-1}$ is friendly. If $x \in s \in \mathcal{K}$ then $\left\{\phi_{k-1}(x)\right\} \cup s \in \mathcal{K}$ by the induction hypothesis and $C \equiv\left\{\delta_{x_{k} \rightarrow y_{k}}\left(\phi_{k-1}(x)\right)\right\} \cup\left(\phi_{k-1}(x) \cup\right.$ $s) \in \mathcal{K}$ since $\delta_{x_{k} \rightarrow y_{k}}$ is a friendly delegation. But $\left\{\phi_{k}(x)\right\} \cup s$ is a subset of $C$, therefore $\left\{\phi_{k}(x)\right\} \cup s$ is a simplex. Thus our claim is proved.

For the converse implication, if $\phi$ is a retraction to $F$ and $E \backslash F=\left\{x_{1}, \ldots, x_{p}\right\}$, we consider $\delta_{x_{1} \rightarrow x_{p}}, \ldots, \delta_{x_{p} \rightarrow y_{p}}$ where $y_{k}=\phi\left(x_{k}\right)$ for $k=1, \ldots, p$. It is easily seen that $\phi=f_{x_{p} \rightarrow y_{p}} \circ \cdots \circ \delta_{x_{1} \rightarrow y_{1}}$. For any $x \in E$ such that $x \neq \phi(x)$ one has $x=x_{k}$ for some $k$, so that $\delta_{x_{\ell} \rightarrow y_{\ell}}(x)=\phi(x)$ if $\ell=k$ and $\delta_{x_{\ell} \rightarrow y_{\ell}}(x)=x$ if $\ell \neq k$. It is easily seen that if $x \in s \in \mathcal{K}$, one has $s \cup \delta_{x_{\ell} \rightarrow y_{k}}(x) \subset s \cup \phi(x)$. It follows that $\delta_{x_{k} \rightarrow y_{k}}$ is friendly if $\phi$ is friendly. 
Corollary 4. $F \subset E$ is a representation if and only if there exists a friendly progressive delegation with outcome $F$.

\section{Topology of the political structure and represen- tations}

Let $\mathcal{K}$ be a simplicial complex on a set $E$ and let $s=\left\{x_{0}, \ldots, x_{r}\right\}$ be a simplex in $\mathcal{K}$. The closed simplex $\bar{s}$ is the set of formal convex combinations $\sum_{i=0}^{r} \lambda_{i} x_{i}$ with $\lambda_{i} \geq 0$ for $i=0, \ldots, r$ and $\sum_{i=0}^{r} \lambda_{i}=1$. Each closed simplex is a metric space under the metric based on Euclidean distance. The geometric realization of $|\mathcal{K}|$ of the simplicial complex $\mathcal{K}$ is the union of all closed simplices $\bar{s}$ for $s \in \mathcal{K}$ endowed with the topology for which $U \subset|\mathcal{K}|$ is open if $U \cap \bar{s}$ is open in $\bar{s}$ for each $s \in \mathcal{K}$. Given simplicial complexes $\mathcal{K}$ on a set $E$ and $\mathcal{L}$ on $F$, a map $\phi: E \rightarrow F$ is simplicial if it takes simplices in $\mathcal{K}$ to simplices in $\mathcal{L}$, that is if $\phi(s) \in \mathcal{L}$ for each $s \in \mathcal{K}$, or, otherwise put, if it extends to a map $\phi: \mathcal{K} \rightarrow \mathcal{L}$. We associate to $\varphi$ the map $|\varphi|:|\mathcal{K}| \rightarrow|\mathcal{L}|$ obtained from $\varphi$ by linear extension.

It is easily seen that friendly retractions are simplicial.

Two simplicial maps $\varphi, \psi:(E, \mathcal{K}) \rightarrow(F, \mathcal{L})$ are said to be contiguous if for any $s \in \mathcal{K}, \phi(s) \cup \psi(s) \in \mathcal{L}$. Denote by $\approx$ the contiguity relation in $E$. $\approx$ is symmetric but generally not transitive. Let $\sim$ be the transitive closure of $\approx$. By definition, $f \sim g$ if there exists a sequence $f=f_{0}, \ldots, f_{p}=g$ such that $f_{k} \approx f_{k+1}$ for $k=0, \ldots, p-1$. Then $\sim$ is transitive and symmetric, hence an equivalence relation, the classes of which are called contiguity classes. Moreover $\approx$ and therefore also $\sim$ are compatible with composition. A simplicial map $\varphi:(E, \mathcal{K}) \rightarrow(F, \mathcal{L})$ is a strong equivalence if there exists $\psi:(F, \mathcal{L}) \rightarrow(E, \mathcal{K})$ such that $\psi \circ \varphi \sim \operatorname{Id}_{E}$ and $\varphi \circ \psi \sim \operatorname{Id}_{F}$. It is worthwhile noting that two simplicial maps that have the same contiguity class are homotopic, but that the converse is not true (cf. e.g. Spanier [14] Chap. 3).

Let $(E, \mathcal{K})$ be a political structure. For any configuration $F$ we define $\mathcal{K}_{F}$ as the set of all elements of $\mathcal{K}$ that are included in $F$. It is clear that $\left(F, \mathcal{K}_{F}\right)$ is a political structure, the agents of which are the elements of $F$. Moreover $\mathcal{K}_{F}$ is a full subcomplex of $\mathcal{K}$. Clearly one has $\mathcal{K}=\mathcal{K}$.

Lemma 5. A map $\varphi: E \rightarrow E$ is contiguous to $\operatorname{Id}_{E}$ if and only if for all $x \in s \in \mathcal{K}$ one has $\{\varphi(x)\} \cup s \in \mathcal{K}$.

Proof. Assume that $\varphi$ has the announced property, and $\left\{x_{1}, \ldots x_{p}\right\}=s \in \mathcal{K}$. Then by induction on $k, s \cup\left\{\varphi\left(x_{1}\right), \ldots, \varphi\left(x_{k}\right)\right\}=s \cup\left\{\varphi\left(x_{1}\right), \cdots, \varphi\left(x_{k-1}\right)\right\} \cup\left\{\varphi\left(x_{k}\right)\right\}$ is in $\mathcal{K}$, therefore $s \cup \varphi(s) \in \mathcal{K}$ and $\varphi$ is contiguous to $\operatorname{Id}_{E}$. Conversely If $\varphi$ is contiguous to $\operatorname{Id}_{E}$ and if $x \in s \in \mathcal{K}$ then $\{\varphi(x)\} \cup s \subset \varphi(s) \cup s \in \mathcal{K}$

In particular we have the following: 
Proposition 6. A retraction is friendly if and only if it is contiguous to $\operatorname{Id}_{E}$.

Remark 1. To a retraction $r$ to $F$ we associate the map $r_{F}: E \rightarrow F$ which is just $r$ with restricted range $F$. If $i: F \rightarrow E$ is the inclusion map, then by definition $r_{F} \circ i=\operatorname{Id}_{F}$. Conversely, any $r_{F}: E \rightarrow F$ such that $r_{F} \circ i=\operatorname{Id}_{F}$ gives rise to the retraction $r$ by putting $r=i \circ r_{F}$. We use same terminology of a retraction to $r_{F}$ when no confusion is possible.
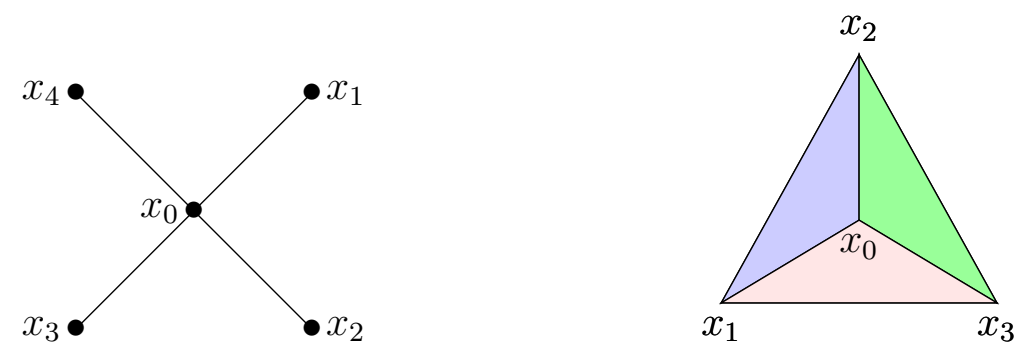

Figure 3: Political structures represented as points connected by lines (1-simplices) or triangles (2-simplices). In both of the above political structures, the retraction onto $x_{0}$ is contiguous to the identity. Consequently $x_{0}$ is a pure R-compromise.

In view of Prop 2 and lemma 5, we can have the following characterization of representations:

Proposition 7. $F$ is a representation of $(E, \mathcal{K})$ if and only if $r_{F}:(E, \mathcal{K}) \rightarrow\left(F, \mathcal{K}_{F}\right)$ is a strong equivalence.

Proof: In view of Lemma $5, r$ is friendly if and only if $r$ is contiguous to $\operatorname{Id}_{E}$, and in view of the Remark 1 this happens if and only if

$$
i \circ r_{F}=r \sim \operatorname{Id}_{E} \text {. }
$$

Since $r_{F} \circ i=\mathrm{Id}_{F}$, this implies that $r_{F}$ is a strong equivalence.

The set of simple friendly delegations in a political structure can be used to define a binary relation $\triangleright$ on $E$ as follows,

$$
x, y \in E, x \triangleright y \text { if either } x=y \text { or the delegation } \delta_{x \rightarrow y} \text { is friendly. }
$$

Lemma 8. For any $x \in E$, let $\sigma_{x}$ be the set of all maximal simplices containing $x$. Then $x \triangleright y$ if and only if $\sigma_{x} \subseteq \sigma_{y}$.

Proof: If $x \triangleright y$ and $x \neq y$, then there is a friendly delegation $\delta_{x \rightarrow y}$, so that every maximal simplex containing $x$ contains $y$ as well. Conversely, if $\sigma_{x} \subseteq \sigma_{y}$, then the map $\delta$ taking $x$ to $y$ and leaving all other elements of $E$ unchanged is a friendly delegation. 
It follows from Lemma 8 that that $\triangleright$ is transitive and reflexive so that $(E, \triangleright)$ is a preordered set.

Definition 5. A representation $F$ of $(E, \mathcal{K})$ is said to be minimal if there is no representation that is a strictly included in $F$

Proposition 9. A representation $F$ of $(E, \mathcal{K})$ is minimal if and only if for any $x, y \in F$, $x \triangleright y$ implies $x=y$.

Proof: If $F$ is not minimal for inclusion then there exists some $F^{\prime} \subset F, x \in F \backslash F^{\prime}$, and $y \in F^{\prime}$ such that $x \triangleright y$. Conversely If there exists $x, y \in F x \neq y$ such that $x \triangleright y$ then $F^{\prime}=F \backslash\{x\}$ is easily seen to be a representation contradicting minimality of $F$.

Lemma 10. Let $\emptyset \neq F \subset E$ be arbitrary.

(i) If $x \triangleright y$ for all $x, y \in F$, then $F \in \mathcal{K}$.

(ii) $E \in \mathcal{K}$ if and only if for any $x, y \in E, x \triangleright y$.

Proof. Assume that $F \notin \mathcal{K}$ and let $s$ be a maximal simplex included in $F$. Since $s \neq F$ there exists some $y \in F, y \notin s$. Let $x \in s$. Since, by the maximality of $s, s \cup\{y\} \notin \mathcal{K}$ one has that $\delta_{x \rightarrow y}$ is not a friendly delegation. This proves (i). (ii) follows immediately from (i)

One may exploit constructions developed for simplicial complexes to obtain a measure of the possibility of delegation. For this purpose, we introduce (cf. Barmak [2]) the nerve $\mathcal{N}(\mathcal{K})$ of $\mathcal{K}$ as the simplicial complex in which the set of vertices is the set of maximal simplices in $\mathcal{K}$, say $\mathcal{K}^{M}$, and the set of simplices is the set of subsets $\left\{s_{0}, \ldots, s_{r}\right\} \subseteq \mathcal{K}^{M}$ such that $\cap_{i=0}^{r} s_{i} \neq \emptyset$. Repeating the construction, one gets $\mathcal{N}^{2}(\mathcal{K})=\mathcal{N}(\mathcal{N}(\mathcal{K}))$ and in generel, $\mathcal{N}^{k}\left(K_{E}\right)=\mathcal{N}\left(\mathcal{N}^{k-1}(\mathcal{K})\right)$

We consider the second nerve $\mathcal{N}^{2}(\mathcal{K})$ in more detail since it plays a role in the characterization of minimal representations. A vertex in $\mathcal{N}^{2}(\mathcal{K})$ is a maximal set $\sigma=$ $\left\{s_{0}, \ldots, s_{r}\right\}$ of maximal simplices in $\mathcal{K}$ such that $\cap_{i=0}^{r} s_{i} \neq \emptyset$. Let $\Phi$ be the set of all maps $\varphi$ from $\mathcal{N}^{2}(\mathcal{K})$ to $E$ assigning to $\sigma=\left\{s_{0}, \ldots, s_{r}\right\}$, a vertex of $\mathcal{N}^{2}(\mathcal{K})$, some element of $\cap_{i=0}^{r} s_{i}$. Notice that the set $\Phi$ is nonempty since $\cap_{s \in \sigma} s \neq \emptyset$ for every $\sigma \in \mathcal{N}^{2}(\mathcal{K})$.

Proposition 11. The set $\Phi$ has the following properties:

(i) Any $\varphi \in \Phi$ is a simplicial map from $\mathcal{N}^{2}(\mathcal{K})$ to $\mathcal{K}$ and an isomorphism onto its image.

(ii) For any $\varphi \in \Phi$ the image $\varphi\left(\mathcal{N}^{2}(\mathcal{K})\right)$ is a minimal representation.

(iii) For each minimal representation $F \subset E$, there is $\varphi \in \Phi$ such that $F=$ $\varphi\left(\mathcal{N}^{2}(\mathcal{K})\right)$.

Proof: (i) Let $\varphi \in \Phi$. If $\left\{\sigma_{0}, \ldots, \sigma_{k}\right\}$ is a simplex in $\mathcal{N}^{2}(\mathcal{K})$, then $\cap_{i=0}^{k} \sigma_{i} \neq \emptyset$, so that there exists a maximal simplex $s$ in $\mathcal{K}$ which belong to all the sets $\sigma_{0}, \ldots, \sigma_{k}$. By its 
definition, $\varphi\left(\sigma_{i}\right)$ belongs to $s$ for $i=0, \ldots, k$, therefore $\left\{\varphi\left(\sigma_{1}\right), \ldots, \varphi\left(\sigma_{k}\right)\right\}$ is a simplex in $\mathcal{K}$. This shows that $\varphi$ is a simplicial map.

Next, we show that $\varphi$ is an injective map. Suppose that $\varphi\left(\sigma_{1}\right)=\varphi\left(\sigma_{2}\right)=x$. If $\sigma_{1}=\left(s_{0}^{1}, \ldots, s_{r}^{1}\right), \sigma_{2}=\left(s_{0}^{2}, \ldots, s_{p}^{2}\right)$, then by our construction $x \in s_{i}^{1}$ for $i=0,1, \ldots, r$ and $x \in s_{i}^{2}$ for $i=0,1, \ldots, p$, so that $\sigma_{1} \cup \sigma_{2}$ is a family of maximal simplices from $\mathcal{K}$ with nonempty intersection. Since $\sigma^{1}$ and $\sigma^{2}$ are already maximal collections of simplices from $\mathcal{K}$ with nonempty intersection, we have that $\sigma^{1}=\sigma^{2}$, so that $f$ is indeed injective.

Finally we prove that the inverse map $\varphi^{-1}$ defined on the image $F$ of $\varphi$ is simplicial. Let $s=\left\{x_{1}, \ldots, x_{k}\right\} \subset F$ be a simplex (i.e. an element of $\left.\mathcal{K}_{F}\right)$ and let $\sigma_{i}=\varphi^{-1}\left(x_{i}\right)$ $i=1, \ldots k$. Denote by $s^{\prime}$ a maximal element of $\mathcal{K}$ containing $s$. For any $i \in\{1, \ldots, n]$, any element of $\sigma_{i}$ has $x_{i}$ as a member, therefore any element of $\sigma_{i} \cup\left\{s^{\prime}\right\}$ has $x_{i}$ as a member. In particular $\sigma_{i} \cup\left\{s^{\prime}\right\}$ is a simplex in $\mathcal{N}(\mathcal{K})$. Since $\sigma_{i}$ is maximal, we have that $s^{\prime} \in \sigma_{i}$. Since every $\sigma_{i} i=1, \ldots, k$, contains $s^{\prime}$ it follows that $\left\{\sigma_{1}, \ldots, \sigma_{k}\right\}$ is a simplex.

(ii) Let $\varphi \in \Phi$ and let $F$ be the image by $\varphi$ of the vertices of $\mathcal{N}^{2}(\mathcal{K})$. For any $x \in E$, let $\sigma_{x}$ be the set of all maximal simplices in $\mathcal{K}$ containing $x . \sigma_{x}$ is thus a simplex of $\mathcal{N}(\mathcal{K})$. If $x \notin F$, let $\sigma$ be a maximal element of $\mathcal{N}(\mathcal{K})$ containing $\sigma_{x}$, and let $y=\varphi(\sigma)$. Then $\sigma_{x} \subset \sigma=\sigma_{y}$, so that by Lemma 8, $x \triangleright y$. This proves that $F$ is a representation. If $x \in F$, there exists $\sigma$ maximal such that $x=\varphi(\sigma)$ so that $\sigma \subset \sigma_{x}$ and therefore $\sigma=\sigma_{x}$. Similarly, if $y \in F$ then $\sigma_{y}$ is maximal and $\varphi\left(\sigma_{y}\right)=y$, but if $x \triangleright y$, then $\sigma_{x} \subset \sigma_{y}$, and by maximality of $\sigma_{x}$ one has $\sigma_{x}=\sigma_{y}$ and therefore $y=x$. It follows from Proposition 9 that $F$ is a minimal representation.

(iii) Conversely, let $F$ be a minimal representation. We claim that for any $y \in F$ the set $\sigma_{y}$ is a maximal element of $\mathcal{N}^{2}(\mathcal{K})$. Suppose not; then there must be $x \neq y$ with $\sigma_{y} \subset \subset \sigma_{x}$. By Lemma 8, $y \triangleright x$, and by Proposition 9, $x \notin F$. But then there must be $z \in F$, such that $x \triangleright z$. By transitivity of $\triangleright$ we get that $y \triangleright z$, and by Proposition 9 $y=z$. It follows that $\sigma_{x} \subset \sigma_{y}$, a contradiction. We conclude that $\sigma_{y}$ is indeed maximal for each $y \in F$.

Remark that for any maximal $\sigma$ one has $\sigma_{x}=\sigma$ for all $x \in \cap_{s \in \sigma} s$. We claim that there exists some $x \in F$ such that $\sigma=\sigma_{x}$. If not then $\sigma=\sigma_{y}$ only for some $y \notin F$. But $y \triangleright z$ for some $z \in F$ and therefore $\sigma \subset \sigma_{z}$ and since $\sigma$ is maximal $\sigma_{z}=\sigma$, a contradiction. In fact such an $x$ is unique since $\sigma_{x}=\sigma_{y}$ where $x, y \in F$ implies $x=y$ (Proposition 9). One therefore may define $\varphi$ by putting $\varphi(\sigma)=x$ where $x$ is the unique $F$ such that $\sigma=\sigma_{x}$. By our construction, $\varphi$ belongs to $\Phi$ and by the preceding paragraph every element of $F$ is an image of some maximal $\sigma$, that is $\varphi\left(\mathcal{N}^{2}(\mathcal{K})\right)=F$.

It follows from proposition 11 that there is a bijection between $\Phi$ and the set of minimal representations of $(E, \mathcal{K})$. 
Example 5 (Simple games). A simple game is a TU game $(N, v)$ (cf. Example 1) with $v(S) \in\{0,1\}$ for all $S \in \mathcal{P}_{0}(N)$, so that it is characterized by the set $\mathcal{W}=$ $\left\{S \in \mathcal{P}_{0}(N) \mid v(S)=1\right\}$ of winning coalitions. Simple games arise in many contexts and has been studied very intensely since their first appearance in [7], see e.g. [13, 8]. There are several ways of associating a political structure with a simple game. The most immediate one is $\left(\mathcal{P}_{0}(N), \mathcal{K}_{v}\right)$ introduced in Example 1 . It is easy to see that $\mathcal{K}_{v}$ is the set of all $\sigma \subset \mathcal{P}_{0}(N)$ such that $\cap_{S \in \sigma \cap \mathcal{W}} S \neq \emptyset$. We are going to show that this political structure is redundant by showing that delegation may lead to trivial representations. Assume that $\mathcal{W} \neq \mathcal{P}_{0}(N)$. If $S_{0} \notin \mathcal{W}$ then any delegation to $S_{0}$ is friendly: actually if $s \in \mathcal{K}_{v}$ then $s \cup\left\{S_{0}\right\}$. It follows that $S_{0}$ is a pure $R$-compromise! One can discard this difficulty by associating to $\mathcal{W}$ the more natural structure $(\mathcal{W}, \mathcal{K})$ where $\mathcal{K}$ the set of all $s \subset \mathcal{W}$ such that $\cap_{S \in \sigma} S \neq \emptyset$. Configurations in $\mathcal{K}$ may be interpreted as situations where a common agreement is possible, whereas conflicts arise when a set $\left\{S_{0}, \ldots, S_{r}\right\}$ is not a simplex. The smallest number $r$ for which this occurs is known as the Nakamura number of $\mathcal{W}$, cf. [6]. Assume $N \in \mathcal{W}$, then any delegation to $N$ is friendly and as a consequence $N$ is an $R$-compromise, and this solution is again trivial ! There are however other political structures that can be associated to $\mathcal{W}$.

(1) Let $\mathcal{W}_{\text {min }} \subset \mathcal{W}$ be the set of winning coalitions which are minimal for inclusion. We assume for simplicity in the following that each $i \in N$ belongs to some $W \in \mathcal{W}_{\text {min }}$. Let $\left(N, \mathcal{K}_{\mathcal{W}}^{1}\right)$ be the political structure with $N$ as set of agents and where a simplex is any nonempty subset of some element of $\mathcal{W}_{\text {min }}$. By our definition, $\mathcal{K}_{\mathcal{W}}^{1}$ is a simplicial complex. A delegation $\delta_{i \rightarrow j}$ in $\left(N, \mathcal{K}_{\mathcal{W}}^{1}\right)$ is friendly when $j$ is in all the minimal winning coalitions containing $i$ (so that $j$ is a partner of $i$ in the terminology of [10]).

(2) In the political structure $\left(\mathcal{W}_{\text {min }}, \mathcal{K}_{\mathcal{W}}^{2}\right)$ the agents are the minimal winning coalitions, and the configurations are sets of coalitions $\left\{S_{0}, \ldots, S_{r}\right\}$ from $\mathcal{W}_{\text {min }}$ such that $\cap_{i=0}^{r} S_{i} \neq \emptyset$.. Note that $K_{\mathcal{W}}^{2}$ is a full subcomplex of $\mathcal{K}$. A delegation from $S$ to $T$ is friendly if for all configurations $\left\{S_{0}, \ldots, S_{r}\right\}$ in $\mathcal{K}_{\mathcal{W}}^{2}$ containing $S$, the configuration $\left\{T, S_{0}, \ldots, S_{r}\right\}$ belongs to $\mathcal{K}_{W}^{2}$. Delegating power to $T$ means that $S$ will not exercise its own power and therefore may be considered as removed from $\mathcal{W}$.

Having dealt with nerves of simplicial complexes in the previous sections, we can see that the poltiical structures (a) and (b) are closely interrelated. Indeed, if $\cup \mathcal{W}_{\text {min }}=N$, then $\mathcal{K}_{\mathcal{W}}^{2}=\mathcal{N}\left(\mathcal{K}_{\mathcal{W}}^{1}\right)$. To see this, we notice that in the nerve of $\mathcal{K}_{\mathcal{W}}^{1}$, vertices are the maximal simplices in $\mathcal{K}_{\mathcal{W}}^{1}$, which are the minimal winning coalitions in $\mathcal{W}$, that is exactly the vertices in $\mathcal{K}_{\mathcal{W}}^{2}$, and simplices are collections of minimal winning coalitions with nonempty intersection, which are the simplices of $\mathcal{K}_{\mathcal{W}}^{2}$.

Irreducible political structures. We end this section by the study of the particular case where no friendly delegations exist.

Definition 6. A political structure $(E, \mathcal{K})$ is irreducible if it has no friendly delegation. 
It is clear that $(E, \mathcal{K})$ is irreducible if and only if it has no nontrivial friendly retract or equivalently that $E$ is a minimal representation in $(E, \mathcal{K})$. The following result can be found in Barmak [2], Chap.5.

Lemma 12. Let $(E, \mathcal{K})$ be irreducible and let $f: E \rightarrow E$ be contiguous to $\operatorname{Id}_{E}$. Then $f=\mathrm{Id}$.

Proof: Let $x \in E$ and let $s$ be a maximal simplex containing $x$. Then $f(s) \cup s$ is a simplex, and $f(x) \in f(s) \cup s=s$, where the last equality follows from the maximality of $s$. Thus, every maximal simplex containing $x$ contains $f(x)$ as well. If $x \neq f(x)$, there would be a friendly delegation $\delta_{x \rightarrow f(x)}$ and $E \backslash\{x\}$ contradicting the irreducibility. We conclude that $f=\operatorname{Id}_{E}$.

The lemma has a useful consequence, which is stated below as a corollary.

Corollary 13. A strong equivalence between two irreducible political structures $\left(F_{1}, \mathcal{K}_{F_{1}}\right)$ and $\left(F_{2}, \mathcal{K}_{F_{2}}\right)$ is an isomorphism.

As a corollary of Proposition 11 we have :

Corollary 14. A political structure $(E, \mathcal{K})$ is irreducible if and only if it is isomorphic to $\mathcal{N}^{2}(\mathcal{K})$.

In partiacular if $(E, \mathcal{K})$ is irreducible then $\Phi$ is a singleton.

\section{Existence and Structure of R-compromises}

In this section we are interested in the structure of R-compromises and the existence of such solutions.

Lemma 15. $Z$ is a representation and if $Z \subset Z^{\prime}$ then $Z^{\prime}$ is representation.

Proof: (i) Let $r$ be a friendly retraction to $Z$, and let $r^{\prime}$ be the map defined by :

$$
r^{\prime}(x)= \begin{cases}r(x) & x \in E \backslash Z^{\prime} \\ x & x \in Z^{\prime} .\end{cases}
$$

Clearly $r^{\prime}$ is a retraction to $Z^{\prime}$. Moreover for any $x \in s \in \mathcal{K}$, either $x \in E \backslash Z^{\prime}$ and $s \cup\left\{r\left({ }^{\prime} x\right)\right\}=s \cup\{r(x)\} \in \mathcal{K}$, or $x \in F$ and $s \cup\left\{r\left({ }^{\prime} x\right)\right\}=s \cup\{x\}=s \in \mathcal{K}$. We conclude that $r$ is friendly.

Remark 2. It is not true that a subset of a R-compromise is a R-compromise. Figure 4 presents an example where there is a represented compromise but where there is no pure represented compromise. 
Corollary 16. Any viable configuration that includes a $R$-compromise is a $R$-compromise.

Let $\mathfrak{R}_{E}$ be the set of all R-compromises and let $R_{E}$ be the set of all pure $\mathrm{R}$ compromises.

Proposition 17. If $R_{E} \neq \emptyset$, then $R_{E} \in \mathcal{K}$ : that is the set of all pure $R$-compromises is a $R$-compromise

Proof: Assume that $R_{E}$ is nonempty, and let $s$ be a maximal (for inclusion) subset of $R_{E}$ that also belongs to $\mathcal{K}$. Let $x \in R_{E}$ be arbitrary, and let $r$ be the retraction to $\{x\}$. Since $r$ is contiguous to $\operatorname{Id}_{E}$, one has that $r(s) \cup s$ is a simplex in $\mathcal{K}$. Since $r(s)=\{x\}$, it follows that $\{x\} \cup s$ is a simplex contained in $R_{E}$. By the maximality of $s$, we have that $x \in s$. Since $x$ is arbitrary we conclude that $s=R_{E}$.

Corollary 18. $R_{E}=E$ if and only if $E \in \mathcal{K}$.

The following result gives some more information about the structure of the $\mathrm{R}$ compromises even in the absence of pure R-compromises.

Proposition 19. Let $\mathcal{I}$ be an arbitrary subset of $\mathfrak{R}_{E}$. Then there exists a subset of $\cup_{F \in \mathcal{I}} F$ which belongs to $\mathfrak{R}_{E}$ and intersects all members of $\mathcal{I}$.

In particular, if $\mathfrak{R}_{E} \neq \emptyset$, then there exists an element of $\mathfrak{R}_{E}$ that intersects all elements of $\mathfrak{R}_{E}$.

Proof: Let $F \in \mathcal{I}$. The collection of simplices included in $\cup_{F^{\prime} \in \mathcal{I}} F^{\prime}$ and containing $F$ is nonempty and has a maximal (for inclusion) element which we denote $c$. Let $F^{\prime} \in \mathcal{I}$ and let $r$ be a retraction to $F^{\prime}$ that is contiguous to $\operatorname{Id}_{E}$, so that $r(c) \cup c$ is a simplex. Since $r(c) \cup c \subset \cup_{F^{\prime} \in \mathcal{I}} F^{\prime}$, it follows from the maximality of $c$ that $r(c) \subseteq c$. Since $\emptyset \neq r(c) \subset F^{\prime}$, we conclude that $c \cap F^{\prime} \neq \emptyset$. Now we prove that $c$ is a friendly retract. Let $r_{c}$ be defined by:

$$
r_{c}(x)= \begin{cases}x & x \in c \\ r_{F}(x) & x \notin c .\end{cases}
$$

If $s \in \mathcal{K}$, then $r_{c}(s) \cup s=r_{c}(s \backslash c) \cup s \subset r_{F}(s) \cup s \in \mathcal{K}$. We conclude that $r_{c}$ is contiguous to $\operatorname{Id}_{E}$, so that $c \in \mathfrak{R}_{E}$.

We now introduce an important concept of the theory of simplicial complexes: The political structure $(E, \mathcal{K})$ is strongly contractible if $(E, \mathcal{K}) \sim\left\{x_{0}\right\}$, for some $x_{0} \in E$; this is equivalent to saying that the identity map on $E$ belongs to the same contiguity class as some constant map. It is interesting to compare this notion with the standard concept of contractibility. $(E, \mathcal{K})$ is contractible if there exists a homotopy from the identity map of $|\mathcal{K}|$ to some constant map. It is known that strong contractibility implies contractibility (cf. [14] Chap. 3, Section 5) but not the converse (cf. [3], Example 2.13).

Proposition 20. If $E$ has a $R$-compromise then $E$ is strongly contractible. 
Proof. Let $x \in F$, where $F$ is a R-compromise. Let $r$ be a contraction to $F$ that is contiguous to $\operatorname{Id}_{E}$, and let $r_{x}$ be the contraction to $\{x\}$, that is $r_{x}$ is the constant map taking all elements in $E$ to $x$. For any $s \in \mathcal{K}, r(s) \cup r_{x}(s) \subset F$, so $r \approx r_{x}$. Since $r \approx \operatorname{Id}_{E}$, one has $r_{x} \sim \operatorname{Id}_{E}$. We conclude that $E$ is strongly contractible.

The converse of Theorem 20 is not true. A configuration may be strongly contractible but fail to have a R-compromise. In the example to the right in Fig.4, no represented compromise exists in $E$, however it is clear that $E$ is strongly contractible.
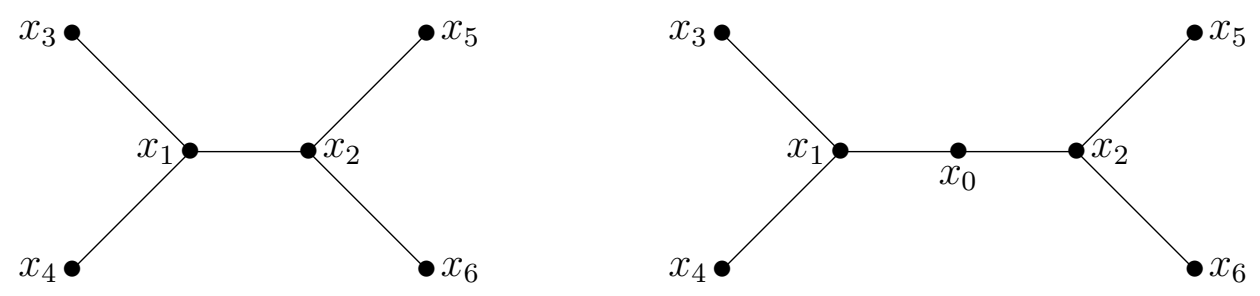

Figure 4: In the political structure to the left there is no pure represented compromise, but the configuration $\left\{x_{1}, x_{2}\right\}$ is a R-compromise. In the political structure to the right no R-compromise exists. Both structures are strongly contractible.

A configuration may have an R-compromise but fail to have a pure R-compromise: In the example to left in Fig.4 neither $\delta_{x_{1} \rightarrow x_{2}}$ nor $\delta_{x_{2} \rightarrow x_{1}}$ are contiguous to identity, so one can verify that no retraction to a singleton exists. Therefore no pure R-compromise exists in $E$. Let $\phi=\delta_{x_{4} \rightarrow x_{1}} \circ \delta_{x_{3} \rightarrow x_{1}}$ and $\eta=\delta_{x_{6} \rightarrow x_{2}} \circ \delta_{x_{5} \rightarrow x_{2}}$. Then $\eta \circ \phi$ is a retraction to $\left\{x_{1}, x_{2}\right\}$. It can be checked that $\phi$ and $\operatorname{Id}_{E}$ are contiguous, and that also $\eta$ and $\operatorname{Id}_{E}$ are contiguous, so that $\eta \circ \phi$ is contiguous to $\operatorname{Id}_{E}$. Therefore $\eta \circ \phi$ is a friendly retraction to $\left\{x_{1}, x_{2}\right\}$, and $\left\{x_{1}, x_{2}\right\}$ is a R-compromise.
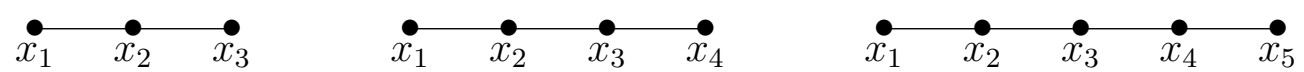

Figure 5: To the left the central point $x_{2}$ is a represented pure compromise; in the middle the central 1-simplex $\left\{x_{2}, x_{3}\right\}$ is an R-compromise and no pure R-compromise exists; to the right there is no R-compromise.

Some other simple cases of political structures are shown in Fig.5. Again it may be noticed that all are strongly contractible but that R-compromises exist only in two of them, and only one has a pure R-compromise. Lemma 11 provides the necessary and sufficient conditions for the existence of an R-compromise. Precisely:

Theorem 21. Let $(E, \mathcal{K})$ be a political structure. The following assertions are equivalent:

(i) There exists a $R$-compromise, 
(ii) One (and therefore any) minimal representation is viable,

(iii) The second nerve $\mathcal{N}^{2}(\mathcal{K})$ is a simplex.

Corollary 22. In a political structure $(E, \mathcal{K})$ a pure $R$-compromise exists if and only if $\mathcal{N}^{2}(\mathcal{K})$ is a singleton.

Example 6 (Networks continued). It is easily seen that an agent with degree 1 can delegate to the only other agent to which she is connected by a configuration. If an agent $x$ with degree $>1$ can delegate to another agent $y$, then by definition any agent connected to $x$ is also connected to $y$; if $z \neq y$ is another agent connected to $x$, then $\{x, z\}$ is viable but $\{y\} \cup\{x, z\}$ is not a viable configuration.

An acyclic connected graph is a tree. For $(E, \mathcal{K})$ be a connected graph, if $(E, \mathcal{K})$ has a pure $\mathrm{R}$-compromise, then $(E, \mathcal{K})$ is a tree where all points except one have degree 1. This follows immediately from the preceding remarks. If $(E, \mathcal{K})$ has an R-compromise, then there are at most two agents $x_{1}, x_{2}$ with degree $\geq 1$, and a $\left\{x_{1}, x_{2}\right\}$ is an $\mathrm{R}$ compromise. Indeed, it is seen immediately that if there are exactly two vertices $x_{1}, x_{2}$ with degree greater than one, then $\left\{x_{1}, x_{2}\right\}$ is a R-compromise. If there are more than two vertices with degree greater than one, then neither of these can delegate to any other. Consequently the case of two vertices with degree $\geq 1$ is the only case which is compatible with the existence of an R-compromise.
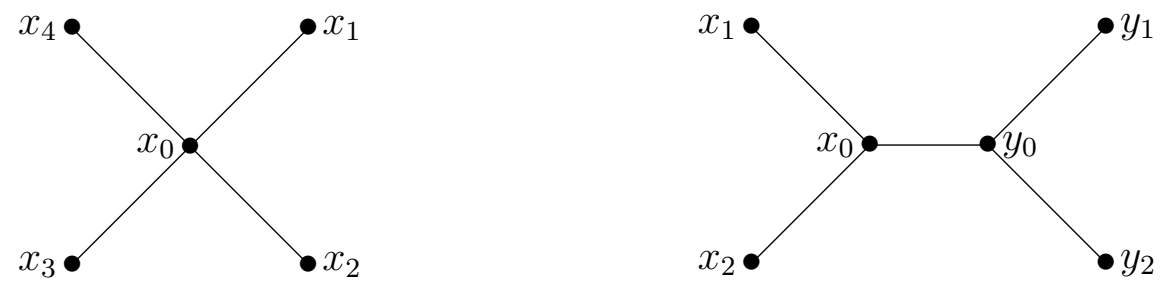

Figure 6: The graph in the left panel represents the class of graphs with a pure R-compromise, and the one in the right panel represents those with an R-compromise containing two agents. Any graphs with a nonempty R-compromise belongs to one of the two classes.

The two cases are illustrated in Fig.6, where the graph to the left has a pure Rcompromise and the graph to the right represents the case where the R-compromise is an edge. It follows from our discussion that except for the number of edges to points with degree 1 , these are the only possible cases of nonempty R-compromises.

\section{Delegated compromise}

In the previous section we have seen that R-compromises, where the agents that are withdrawn from the political contentions remain represented by suitable representatives, 
in the sense that the representing agent enters into all the viable configurantions where the agent who withdrew might have been present. Unfortunately, R-compromises may not always exist; indeed we saw that the existence of R-compromises imply that the political structure, viewed as a simplicial complex, is strongly contractible, in itself a rather restrictive property, but this was not enough, since even a strongly contractible political structure may fail to exhibit R-compromises.

This shortcoming has to do with the restrictions that we have put on a delegation. In an R-compromise the delegation $\delta_{x \rightarrow y}$ amounts to removing $x$ from the outcome, but it does not imply the withdrawal of $x$ from the search for a compromise. This happens since any other other delegation $\delta_{x^{\prime} \rightarrow y^{\prime}}$, in order to be friendly, must secure that $y^{\prime} \cup s$ be viable for all $s$ that contain $x^{\prime}$, and the simplex $s$ may contain $x$. In this sense $x$ is not totally absent from the seach for a compromise even after the delegation.

In the following we present a slightly weaker notion called delegated compromise, in which a delegation from $x$ to $y$ implies that $x$ withdraws from the process, so that further delegations need not take $x$ into account. This clearly makes the search for a solution easier.

Definition 7. A configuration $F$ of $(E, \mathcal{K})$ is a narrowing of $E$ if there exists a decreasing sequence $E=F_{0} \supset F_{1} \supset \cdots \supset F_{n}=F$ and friendly retractions $r_{i}$ of $\left(F_{i-1}, \mathcal{K}_{F_{i-1}}\right)$ onto $F_{i}$ for $i=1, \ldots, n$

Notice that the retraction $r_{i}$ are relative to the simplicial complex $\left(F_{i-1}, \mathcal{K}_{F_{i-1}}\right)$. It follows from Proposition 7 that this is equivalent to require that the associated $\hat{r}_{i}:\left(F_{i-1}, \mathcal{K}_{F_{i-1}}\right) \rightarrow\left(F_{i}, \mathcal{K}_{F_{i}}\right)$ is a strong equivalence for $i=1, \ldots, n$. Therefore we have the following:

Proposition 23. If $F$ is a narrowing of $(E, \mathcal{K})$ then $\left(F, \mathcal{K}_{F}\right)$ is strongly equivalent to $(E, \mathcal{K})$.

In view of Proposition 3 any narrowing $F$ can be obtained by a progressive delegation $\delta_{x_{1} \rightarrow y_{1}}, \ldots, \delta_{x_{p} \rightarrow y_{p}}$ such that $\delta_{x_{p} \rightarrow y_{p}} \circ \cdots \circ \delta_{x_{1} \rightarrow y_{1}}(E)=F$ and such that the restrictions of $\delta_{x_{k} \rightarrow y_{k}}$ to $E \backslash\left\{x_{1}, \ldots, x_{k-1}\right\}, k=1, \ldots, p$, are friendly. We are ready to introduce the main concept of solution of this section:

Definition 8. A configuration $F$ of $(E, \mathcal{K})$ is a delegated compromise (D-compromise for short) if $F$ a narrowing of $(E, \mathcal{K})$ and $F \in \mathcal{K}$.

The obvious way of looking at delegated compromises is to see them as repeated compromises; in the first step, $F_{1}$ is an R-compromise for $E$, in the next step an R-compromise $F_{2}$ in $F_{1}$ is obtained, etc. It should be noted however, that the sets $F_{i}$ themselves need not be R-compromises in the original structure since we do not demand that they belong to $\mathcal{K}$. It is clear that an R-compromise is a delegated compromise, but 
the converse need not be true. Note that a D-compromise must be a viable configuration (a simplex).

The D-core. Given a political structure, one can try to search for a D-compromise following a process of successive friendly delegations, but is there a narrowing that leads to a positive outcome, namely a viable configuration? In order to capture the best narrowing that can be obtained from such a search, we need to know what are the minimal configurations that can be achieved as outcome.

The idea of best narrowing is captured by the following:

Definition 9. A configuration $F$ of $(E, \mathcal{K})$ is a delegated core (shorthand: a D-core ${ }^{1}$ ) of $(E, \mathcal{K})$ if $F$ is a narrowing of $(E, \mathcal{K})$ and $\left(F, \mathcal{K}_{F}\right)$ is irreducible.

Now we can state and prove the main result about D-cores of political structures:

Proposition 24. Let $(E, \mathcal{K})$ be a political structure. Then the following hold:

(i) $(E, \mathcal{K})$ has a D-core,

(ii) $(E, \mathcal{K})$ is strongly equivalent to its D-core,

(iii) the D-core is unique up to isomorphism.

Proof: (i) Either $(E, \mathcal{K})$ is irreducible, in which case it is itself a D-core, or one can find a non trivial friendly retract $F_{1}$. If $F_{1}, \mathcal{K}_{F_{1}}$ is irreducible, otherwise we repeat the operation on $\left(F_{1}, \mathcal{K}_{F_{1}}\right)$ and so on we thus construct a narrowing $E=F_{0} \supset F_{1} \supset \cdots \supset$ $F_{k} \supset \cdots$. Since the sequence is strictly decreasing and $E$ is finite we reach eventually an index $n$ with irreducible simplicial complex $\left(F_{n}, \mathcal{K}_{F_{n}}\right)$. Clearly this is a D-core. (ii) follows from Proposition 23. It remains only to prove (iii): Suppose that $\left(F_{1}, \mathcal{K}_{F_{1}}\right)$ and $\left(F_{2}, \mathcal{K}_{F_{2}}\right)$ are two D-cores of $(E, \mathcal{K})$. Then by (ii) we have that

$$
\left(F_{1}, \mathcal{K}_{F_{1}}\right) \sim(E, \mathcal{K}) \sim\left(F_{2}, \mathcal{K}_{F_{2}}\right)
$$

and by the Corollary to Lemma $12,\left(F_{1}, \mathcal{K}_{F_{1}}\right)$ and $\left(F_{2}, \mathcal{K}_{F_{2}}\right)$ are isomorphic.

Using the D-core as a formal description of conflict resolution may seem attractive in view of these results. On the other hand, it should be remembered that the $\mathrm{D}$-core does not point to a single configuration, rather it is an indication of the limits beyond which conflicts cannot be solved by delegation alone.

Existence of D-compromises. We now investigate the existence of a D-compromise in a political structure. By definition, if the D-core is viable, then it is a D-compromise. The D-core always exists (Proposition 24); on the other hand, being irreducible, if it is viable it must be a singleton. These considerations are made precise by the following propostiion.

\footnotetext{
${ }^{1}$ The term "core" is used by Barmak [2], Chap.5, for the same notion in the context of abstract finite spaces.
} 
Proposition 25. Let $(E, \mathcal{K})$ be a political structure. Then the following hold:

(i) $(E, \mathcal{K})$ has a D-compromise,

(ii) $(E, \mathcal{K})$ has a pure D-compromise,

(iii) $(E, \mathcal{K})$ is strongly contractible,

(iv) The D-core is a singleton.

Proof: (i) $\Leftrightarrow($ ii). If $F$ is a D-compromise, then, since $F$ is a simplex, there exists a friendly retraction to any of its points $\{x\}$. It follows that $\{x\}$ is a narrowing of $(E, \mathcal{K})$ an therefore that $\{x\}$ is a pure D-compromise.

(ii) $\Rightarrow$ (iii). Since $\{x\}$ is a narrowing, we have that $(E, \mathcal{K}) \sim\{x\}$, so that $(E, \mathcal{K})$ is strongly contractible.

(iii) $\Rightarrow($ iv $)$. Let $F$ be a D-core. From $(E, \mathcal{K}) \sim\left(F, \mathcal{K}_{F}\right)$ and $(E, \mathcal{K}) \sim\{x\}$, we get that $\left(F, \mathcal{K}_{F}\right) \sim\{x\}$. Since both $\left(F, \mathcal{K}_{F}\right)$ and $\{x\}$ are irreducible, they are isomorphic by the Corollary to Lemma 12 .

(iv) $\Rightarrow$ (ii) is straightforward, since the D-core being a singleton is also a pure Dcompromise.

We may exploit the results on nerves of simplicial complexes obtained earlier to obtain additional information about narrowings of the political structure.

If $(E, \mathcal{K})$ is not irreducible, $\mathcal{N}^{2}(\mathcal{K})$ is not isomorphic to $(E, \mathcal{K})$, but the number of vertices in $\mathcal{N}^{2}(\mathcal{K})$ is no greater than that of $(E, \mathcal{K})$, and one may proceed to the derived nerves $\mathcal{N}^{k}(\mathcal{K})$ ) for $k>3$. Clearly, there will be some number $d \geq 1$ such that $\mathcal{N}^{2 d+2}(\mathcal{K})$ and $\mathcal{N}^{2 d}(\mathcal{K})$ have the same number of vertices. By convention $\mathcal{N}^{0}(\mathcal{K})=\mathcal{K}$.

Definition 10. Let $(E, \mathcal{K})$ be a political structure. The depth of delegation of $(E, \mathcal{K})$, written $d_{(E, \mathcal{K})}$, is the smallest number $d$ such that the $\mathcal{N}^{2 d+2}(\mathcal{K})$ and $\mathcal{N}^{2 d}(\mathcal{K})$ have the same number of vertices.

Using Lemma 11, we get the following proposition.

Proposition 26. $\mathcal{N}^{2 k}(\mathcal{K})$ is isomorphic to the D-core of $(E, \mathcal{K})$ if and only if $k \geq d_{(E, \mathcal{K})}$.

Clearly the depth provides the smallest number of iterated representations in a narrowing to achieve the D-core.

In the remaining part of this section, we consider a political structure which is a graph, as considered in Example 2. We saw the existence of a pure R-compromise had very far-reaching consequences. With delegated compromises, the situation is quite different. Here the existence of a delegated compromise has rather far-reaching consequences.

Proposition 27. Let $(E, \mathcal{K})$ be a political structure which is a graph. The following are equivalent:

(i) $(E, \mathcal{K})$ is a tree, 
(ii) each $x \in E$ is a pure delegated compromise in $(E, \mathcal{K})$,

(iii) the simplicial complex $\mathcal{K}$ is strongly contractible,

(iv) the topological space $|\mathcal{K}|$ is contractible.

Proof: (i) $\Rightarrow$ (ii). Let $x \in E$ be arbitrary, and for each $y \in E$, define the distance from $y$ to $x, d(y, x)$, as the number of edges in a path from $x$ to $y$; since $(E, \mathcal{K})$ is a tree, $d(x, y)$ is well-defined. Let $D=\left\{x_{1}, \ldots, x_{k}\right\} \subset E$ be the set of all vertices in $E$ with maximal distance from $x$; then each $x \in D$ has degree 1 . Let $E_{1}=E \backslash D$. Each $x \in D$ is connected to some $y(x) \in E$ which does not belong to $D$ since $(E, \mathcal{K})$ is a tree. There is a friendly delegation $\delta_{x \rightarrow y(x)}$ from $x$ to the vertex $y(x)$, and the composition $\delta_{x_{k} \rightarrow y\left(x_{k}\right)} \circ \cdots \circ \delta_{x_{1} \rightarrow y\left(x_{1}\right)}$ is progressive and defines a narrowing from $(E, \mathcal{K})$ to $\left(E_{1}, \mathcal{K}_{E_{1}}\right)$. The political structure $\left(E_{1}, \mathcal{K}_{E_{1}}\right)$ is a graph which is connected and has no cycles, hence it is a tree. Moreover $E_{1}$ has strictly less elements than $E$. Repeating the above procedure, we successively obtain substructures $\left(E_{i}, \mathcal{K}_{E_{i}}\right)$ and narrowings $\left(E_{i}, \mathcal{K}_{E_{i}}\right) \rightarrow\left(E_{i+1}, \mathcal{K}_{E_{i+1}}\right)$, for $i=1, \ldots, p$, untill we obtain a structure $\left(E_{p+1}, \mathcal{K}_{E_{p+1}}\right)$ in which no agent has degree 1 . But a tree without vertices of degree 1 consists of the point $x$, which consequently is a delegated compromise of $(E, \mathcal{K})$.

(ii) $\Leftrightarrow$ (iii) is a consequence of Theorem 25 , (iii) $\Rightarrow$ (iv) is a consequence of Lemma 2 in Chapter 3, Section 5 in Spanier [14], and finally (iv) $\Rightarrow(\mathrm{i})$ is Lemma 1 in Chapter 3, Section 7 in Spanier [14].

It is seen that delegated compromises exist if and only if the political structure is strongly contractible. The topology of the political structure, and precisely its strong homotopy, is the basic determinant for the possibility of a compromise. When delegated compromises exist, they may take the form of single agents (pure D-compromise) or a viable configuration. The question whether some D-compromise may seem unacceptable (dictatorial) is irrelevant in our setting. This is due to the fact that in our model we are concerned only by the viability of the solution. In this sense the model may look underdetermined: It may be the case that the political environment requires some further conditions for viable configurations to be acceptable, as seen in the empirical case in Example 4, where the confessional composition of any compromise is an indispensable pre condition which must be satisfied by a viable configuration. Future research must therefore consider viability with some formal kind of acceptability.

\section{Concluding remarks}

In the present paper, we have presented a formal model of compromise in contexts of political decision making. This model is that of a political structure, mathematically a simplicial complex in which vertices are interpreted as political agents and simplices as viable configurations. In this context, the fundamental motive of action is founded on the notion of delegation. Delegation can be formulated as a mapping taking the 
delegating agent to another agent, to whom power is to be transferred, and the idea of handling over influence to another agent is formalized through the notion of friendliness. The analysis of delegation and its consequences can now exploit the theory of maps between simplicial complexes, and due the discrete character of the model the relevant mathematics appear to be that of strong homotopy.

Two fundamental types of delegation are of interest. In the first one, influence may be transferred but the receiver must be able to respond back and cannot delegate further. This gives rise to the notions of representations and R-compromises. Allowing for repeated delegation opens up for further concentration of power, expressed by notions of D-cores and D-compromise.

Since a general theory must be assessed in terms of the insights, which it offers in particular applications, we have briefly considered some such applications of the theory, namely to TU and in particular simple games, to effectivity functions, and to network models of political influence. The notion of delegation indicates how to analyse situations where decisions are not immediately obtainable so that compromises are called for. We have only touched upon this area of research where there are several directions of future developments of the theory. One of such directions is to consider situations where acceptability conditions in addition to viability are required for a compromise.

The approach to political structures through simplicial complexes may be applied to other aspects of decision making than delegation, as it has been done in the present paper. Instead of power being handed over from some agents to other, one may consider the exclusion of some agents by the others, a process which can also be treated using suitable notions of homotopy. This again is a topic of future research.

\section{References}

[1] Abdou, J. and H.Keiding (1991). Effectivity functions in social choice. Kluwer, Dordrecht.

[2] Barmak, J.A. (2011), Algebraic topology of finite topological spaces and applications, Springer, Heidelberg.

[3] Barmak, J. A., and E.G.Minian (2012). Strong homotopy types, nerves and collapses. Discrete and Computational Geometry, 47(2), 301-328.

[4] Moulin, H. and B.Peleg (1982). Cores of effectivity functions and implementation theory. Journal of Mathematical Economics 10, 115-145.

[5] Freeman, L.C. (1978). Centrality in social networks: conceptual clarification, Social Networks 1, 215-239. 
[6] Nakamura, K. (1979). The vetoers in a simple game with ordinal preferences, International Journal of Game Theory 8, 55-61.

[7] von Neumann, J. and O.Morgenstern (1944). Theory of games and economic behavior, Princeton.

[8] Peleg, B. (1984). Game theoretic analysis of voting in committees, Cambridge University Press, Cambridge.

[9] (2010). Strategic social choice: Stable representations of constitutions. Springer, Heidelberg.

[10] Reny, P.J. and M.H.Wooders (1996), The partnered core of a game without side payments, Journal of Economic Theory 70, 298-311.

[11] Schmitt, C. (2008). The concept of the political: Expanded edition. University of Chicago Press.

[12] Scott, J. (2011). Social network analysis: developments, advances, and prospects. Social Networks Analysis and Mining 1, 21-26.

[13] (1962). Simple games: An outline of the descriptive theory. Systems Research and Behavioral Science 7, 59-66.

[14] Spanier, E.H. (1966). Algebraic topology, McGraw-Hill, New York. 